\title{
Latino fathers report low awareness and knowledge of the human papillomavirus vaccine, but high willingness to vaccinate their children if recommended by primary healthcare provider: a qualitative study
}

\author{
Ana Cristina Lindsay 1,*, Denisse Delgado 2, Madelyne J. Valdez ${ }^{1}$, and Phillip Granberry ${ }^{3}$ \\ 1 Department of Exercise and Health Sciences, College of Nursing and Health Sciences, University of \\ Massachusetts Boston 1; Ana.Lindsay@umb.edu (ORCID\#: 0000-0002-2520-0493); \\ Madelyne.Valdez001@umb.edu \\ 2 Department of Public Policy and Public Affairs, McCormack Graduate School of Policy and Global Studies, \\ University of Massachusetts Boston; D.Delgadovazquez@umb.edu \\ 3 Department of Economics, College of Liberal Arts and The Mauricio Gastón Institute for Latino \\ Community Development and Public Policy, University of Massachusetts Boston; \\ Phillip.Granberry@umb.edu (ORCID\#: 0000-0001-9280-2016) \\ * Correspondence: Ana.Lindsay@umb.edu; Tel.: 1-617-287-7579 (A.C.L.)
}

\begin{abstract}
Objective: Despite increasing interest in understanding factors influencing awareness, knowledge, and acceptability of the human papilloma virus (HPV) vaccine among Latino parents to date, limited information is available specific to Latino fathers living in the United States. Methods: This descriptive qualitative study explored Latino fathers' awareness, knowledge, and acceptability of the HPV vaccine for their adolescent children. Data were collected through individual, semi-structured interviews and analyzed using a hybrid method of thematic analysis that incorporated deductive and inductive approaches. Results: Nineteen, majority foreign-born Latino fathers $(63.2 \% ; n=12)$ fathers of male and female adolescents participated in the study. Four main themes and two subthemes emerged from the analyses. Results found fathers' low awareness and knowledge of HPV and the HPV vaccine. Results also identified fathers' positive attitude toward vaccines in general. Moreover, results revealed fathers' trust in healthcare providers. This trust translated into an increased willingness to vaccinate their children against HPV if recommended by their child's primary healthcare provider. Conclusion: Findings indicate the need for increased efforts to raise awareness and knowledge among Latino fathers of HPV and the HPV vaccine. In addition, findings underscore the critical role of healthcare providers' recommendation of the HPV vaccine. Given the limited research focused on Latino fathers, this study's findings are valuable in building a knowledge foundation needed for developing future studies and interventions to promote the HPV vaccine by targeting Latino fathers living in the United States. Future research should quantify Latino fathers' awareness, knowledge, and acceptability of the HPV vaccine for their children, and preferences for educational interventions to promote HPV vaccination.
\end{abstract}

Keywords: Fathers; Human papillomavirus (HPV); awareness; HPV knowledge; HPV vaccine acceptability; Latino; Hispanic men 


\section{Introduction}

Human papillomavirus (HPV) is the most common sexually transmitted disease in the United States, and an estimated 14 million persons are newly infected with HPV every year [1]. HPV infection has cancer-related consequences for both men and women [1,2]. HPV infection is etiologically linked to several types of cancers including those of the cervix, vulva, vagina, penis, anus, and oropharynges, thus representing a significant public health concern $[1,2]$.

Vaccination against HPV is the optimal way to prevent HPV-associated cancers conferring protection from the most common types of HPV that can cause cervical, oropharynges, vulvar, vaginal, penile, and anal cancers $[1,2]$. In the United States, the HPV vaccine has been recommended for girls at age 11 or 12 years (although the series can be started at age 9 years) since 2006, and also through age 26 years for women not vaccinated previously. In 2011, with additional scientific evidence from clinical trials, routine HPV vaccination was recommended for boys aged 11 or 12 years and for those through 21 years not vaccinated previously [2]. The target age range for HPV vaccination is based on evidence that the vaccine is most effective before any exposure to HPV which is sexually transmitted and also to facilitate administration with two other recommended vaccines (i.e., tetanus-diphtheria-acellular pertussis [Tdap] and meningococcal vaccines [MenACWY]) the same age range [3]. The vaccine series initially included 3 doses. However, in 2016 with accumulated evidence of comparable antibody response to 3 doses, a revised recommendation for a 2-dose series, six-months apart was issued by the Advisory Committee on Immunization Practices (ACIP), the Centers for Disease Control and Prevention (CDC) [3,4].

Although HPV vaccination is a safe and effective primary prevention strategy, its uptake rate remains low in the United States [3,4]. In 2018, data from the 2018 National Immunization SurveyTeen (NIS-Teen) showed that despite improvement from previous years, only $51.1 \%$ of adolescents aged 13-17 were up to date with the recommended HPV vaccination series, well below the $80 \%$ target set by the Healthy People 2020 and lagging behind the coverage for the other two vaccines - Tdap at $89 \%$ and MenACWY at $86.6 \%[4,5]$.

Evidence indicates low-income, minority, and immigrant groups are at increased risk of HPVassociated cancers [6-9]. Low or inadequate knowledge and awareness of HPV infection and the HPV vaccine have been linked to increased HPV-associate cancer risk among minorities [6-11].

Latinos are the largest minority population in the United States, and evidence indicates higher HPV-associated cancer incidence and mortality among Latinos compared to non-Hispanic whites $[12,13]$. Despite an increasing number of studies conducted to identify HPV awareness and acceptability of the HPV vaccine among Latinos [14-21], only very limited research is specific to Latino fathers [22-25]. The extant literature has mainly focused on Latina mothers' awareness, acceptability, and uptake of the HPV vaccine for their adolescent children, leaving fathers underrepresented in HPV research [14-21].

Although evidence suggests that mothers are the parent most likely to take their children to health care visits, fathers play a central role in the Latino family. Evidence suggests their influential role in the health care of their children $[22,23,26]$. Therefore, the current study was designed to explore Latino fathers' awareness, knowledge, and acceptability knowledge and of the HPV vaccine for their adolescent children.

\section{Methods}

\subsection{Study Design and Sample}

This study received approval from the Institutional Review Board (IRB) at the University of Massachusetts Boston (IRB \# 2017221). Using an exploratory descriptive qualitative methodology, we conducted one-on-one, face-to-face, semi-structured interviews with Latino fathers. Fathers were eligible to participate if they: (1) had at least one child aged 11-19 years; (2) self-identified as Latino; (3) lived in Massachusetts; (4) lived with or had shared parental responsibility to the said child; and (5) provided signed informed consent.

We used purposive sampling methodology to recruit a convenience sample of Latino fathers through social networking, a "word of mouth" or snowball sampling approach. This involved two 
strategies: (1) leveraging the personal and community networks of the Latino research staff to identify potential participants, and then recruit them for the study [27], and (2) asking early enrollees to recommend their Latino friends who had at least one child aged 11-19 years [27]. Research team members screened interested individuals in-person or via telephone. Those who met the eligibility criteria were asked to participate in the study. Team members scheduled interviews with eligible participants at a convenient time and location (i.e., either at participant's home, private offices at the academic institution, or in public settings including s libraries or coffee shops). After obtaining written and verbal informed consent, staff conducted interviews in the participant's preferred language (Spanish or English).

\subsection{Data Collection}

Face-to-face individual qualitative interviewing with eligible participants was conducted by two trained, bilingual (native Spanish-speaker and English) Latina research assistants. They used a semistructured interview guide to ensure that similar topics were discussed with all participants [28]. The interview guide included open-ended questions based on available literature and was designed to elicit participants' perceptions and understanding of HPV infection, HPV-associated cancers, and the HPV vaccine [20,29-31]. In addition, interviewers asked follow-up questions and used targeted probes to clarify information and/or collect additional relevant information based on information shared by each participant. The interview also included a brief, close-ended questionnaire on participants' socio-demographic information and acculturation level assessed via the Short Acculturation Scale for Hispanics (SASH), a 12-item measure scale validated for use in Latino populations [32,33].

The guide was piloted-tested with one Latino father, and data from the pilot interview was not included in the present study, but the results were used to refine the guide prior to use in the subsequent interviews. Before each interview, the interviewers (DDV, MJV) asked participants' preferred language for conducting the interview. Following, interviewers explained the study's purpose and interview procedures, including confidentiality of information in the participant's preferred language and obtained written informed consent. Participants who had more than one child between the ages of 11 and 19 years were asked to share information about each child. A trained, bilingual research assistant (MJV) took notes during all interviews. The interviews were audiorecorded after obtaining verbal consent from participants and lasted approximately 25-35 minutes. Participants received a $\$ 25$ gift card for their participation.

\subsection{Data Analysis}

Data were analyzed using standard qualitative methods [28]. All audio-records were transcribed verbatim in Spanish or English by a bilingual, native Spanish speaker, professional transcriptionist within a few days of the interview [28,34]. Data collection and preliminary data analysis were undertaken concurrently $[28,34]$. The interviewer and research assistant met for about 20 minutes after each interview to discuss recurring themes and identify new additional themes.

Once all interviews were completed and transcribed, the transcripts were analyzed using thematic analysis, an iterative multi-phase process of coding the data in phases to create meaningful patterns by two experienced bilingual qualitative researchers (ACL, DDV). First, two experienced qualitative researchers and bilingual speakers (ACL, DDV) read several transcripts numerous times to become familiar with the content and generate initial codes [28,34]. Following, the researchers manually coded all transcripts independently using a hybrid approach of thematic analysis that incorporated an iteration of 1) deductive approach including a priori themes based on the interview guide and literature, and 2) data-driven inductive approach that allowed for new themes to emerge during analysis, thus expanding the possible a priori categories of themes $[28,34]$. Throughout this phase, the two researchers met regularly to discuss coding and to identify and resolve coding disagreements [34]. Coded text describing similar ideas were grouped and sorted to identify emergent themes and subthemes. Finally, salient text passages were extracted and translated into English as illustrative quotes for emergent themes. In addition, descriptive statistics and frequencies were calculated for data collected in the socio-demographic survey using Microsoft Excel 2008. 


\section{Results}

\subsection{Sample}

A total of 19 fathers participated in this study. As shown in Table 1, approximately 63\% were foreign-born $(63.2 \% ; n=12)$ fathers of adolescent boys $(\sim 53 \% ; n=10)$ and girls $(\sim 74 \% ; n=14)$ aged between 11 and 19 years (mean age of adolescents: 15.3 years). A little more than half $(57.9 \% ; n=11)$ of the fathers were of Caribbean (Puerto Rico; $n=6$, and Dominican Republic; $n=5$ ) descent, whereas approximately $26 \%(26.3 \% ; n=5)$ were South American (Colombia; $n=4$, and Peru, $n=1)$, and about $11 \%(10.6 \% ; n=2)$ Central American (El Salvador; $\mathrm{n}=1$, and Guatemala; $\mathrm{n}=1)$. The majority $(84.2 \% ; n=16)$ chose to conduct the interview in Spanish and approximately $78.9 \%(n=15)$ reported low acculturation levels (SASH < 2.99). Table 1 presents additional sample characteristics.

We identified four main themes: (1) low awareness of HPV and the HPV vaccine; (2) low knowledge of HPV infection and HPV-associated cancer risk for chidren; and (3) positive attitudes toward vaccines in general; and (4) high willingness to have their children (boys and girls) vaccinated against HPV if recommended by their children's primary healthcare provider. These themes are discussed below with illustrative quotes from participants.

\subsection{Themes and Subthemes}

3.2.1. Theme: Low awareness of HPV and the HPV vaccine

A little less than half of the fathers $(n=9 ; 47.4 \%)$ knewabout HPV. Most fathers reported hearing about HPV from media (television and social media - Facebook) and personal (e.g., family members, mostly partner and friends) sources. Only a couple of fathers $(n=2 ; 10.5 \%)$ mentioned hearing about HPV from healthcare providers. These are illustrated in the following two quotes:

Yes, I have heard about HPV. The scientific name I really don't know what it means, but I believe the $H P V$ is linked to having sexual relations. I mean the transmission is from maintaining relationships with different people without protection... I heard on TV and my cousin in Peru had her daughter take the vaccine. She told me was to prevent cervical cancer which her mother (grandmother) had it, so she felt it was good to give to her daughter because one never knows it could run in the family, right? After that I was curious, so I searched on the Internet. That's how I learned about it.

I didn't really hear about it, but my wife told me that last year when my daughter was at the doctor they asked if she would allow them to give [daughter] the vaccine and she said yes, and they put one dose and told her she needs to go back after a few months and they will put one more dose... when she got home, she told me, listen [daughter's name] got vaccine shots today... I don't oppose to any vaccine.

Furthermore, some fathers $(n=5 ; 26.3 \%)$ appeared to confuse HPV with HIV. When reminded that HPV was not the same of HIV, some fathers then reported not knowing about HPV, while a couple pondered if HPV was also a sexually transmitted disease.

No, never heard about papilloma virus. You said HPV, right? No, not this word I never heard of it. I heard of HIV, but not HPV. This is the first time I hear about it when you asked me. I have heard a lot about HIV, but not HPV. It sounds similar... could it be related to sexual relations? I really have not heard about it before.

Less than half $(n=6 ; 36.8 \%)$ of the fathers were aware of the HPV vaccine. The majority of the fathers who were aware of the HPV vaccine ( $n=5$ of $7 ; 71.4 \%$ ) believed that only girls should be vaccinated. One father said,

Look, I don't know much about this vaccine. I heard in passing on a Hispanic show on TV, but I didn't pay much attention and I don't really know much about it. What I think is that it's a vaccine that girls get. I would have to ask my wife to know if my daughter got it. I wouldn't be able to tell you... 
Only a couple of fathers ( $\mathrm{n}=2$ of $7 ; 28.6 \%$ ) believed that boys should be vaccinated. For example, one father mentioned:

At first, I did not know boys should also receive this vaccine [HPV]. I thought it was only for girls because I thought it was to prevent cervical cancer. But when my wife took my son to his last doctor appointment just after he had turned 11 [years], the doctor told my wife about the HPV vaccine. My wife was surprised too because she also did not know that boys need this vaccine. My daughter already had the vaccine, so my wife said yes and that's how I know that boys also should get this vaccine...

Moreover, when discussing their beliefs about the HPV vaccine, the majority of fathers ( $\mathrm{n}=17$, $89 . .5 \%$ ) expressed uncertainty and often referred to their child's mother or partner as the main parent taking care of children's health. These are illustrated in the following quotes.

Mostly my wife takes care of those things [vaccination]. Now when it's something more serious, say an appointment for something serious, like when the older one was hurt in a motorcycle accident, things like that, they include me. And I'm there to support them, my family, when they need me. But normally the mom takes care of those things [vaccination] in our family...

Perhaps I should be more informed, but the truth is that thank God the mother of my children is a wonderful woman and I do not have to worry about my children, their medical appointments, things like that, they are well cared for [by the mother].

My wife is the one who always takes the kids to the doctor, but she keeps me informed...

3.2.2. Theme: Low knowledge of HPV transmission and HPV-associated cancers

The majority of fathers reported low knowledge about HPV transmission. Less than half of the fathers $(n=8 ; 42.1 \%)$ were aware of HPV being a sexually transmitted disease.

I don't know too much about it but I know it's transmitted by sexual relations and that if one is not cautious about protection like using condoms or one has many partners and have unprotected relations [sex] one can get the virus...

Interviewers further probed fathers who were aware of HPV $(n=11 ; 57.9 \%)$ about the link between HPV and HPV-associated cancers. Most fathers ( $\mathrm{n}=7$ of $11 ; 63.6 \%)$ mentioned HPV being associated with cervical cancer. Only two fathers $(2$ of $11 ; 18.2 \%)$ discussed HPV being associated with other types of cancers including cancer of the throat and anus. Moreover, a salient finding was that all fathers who reported some knowledge of HPV transmission and its association with cervical cancer reported having a family member or a friend who had been affected by cervical cancer. A couple of fathers said,

I know this virus can cause cervical cancer. I know this because my mother and my older sister had this cancer. But thank be to God, they [doctors] were able to treat them. For my mother was a bit worse [cervical cancer] and she suffered a lot, but for my sister was not as bad and she recovered quickly. Thank to the Lord and the doctors they both survived because they discovered the cancer and treated them...

I know a guy in my hometown back in the Dominican Republic who had cancer on the throat and what I heard from others is that it was because of this virus... rumor is that he despite having a family he was out there with many women and lots of prosmicuity. When he got sick apparently the doctor said it was this virus, HPV. People thought he had HIV, but no. He had to have a surgery in the throat. He survived but I don't know much about him since I came to the United States.

3.2.3. Theme: Positive attitude towards vaccines in general 
Despite fathers' low knowledge of the HPV vaccine, the majority $(n=16,78.9 \%)$ reported positive attitude toward vaccines in general. Most fathers discussed their personal and their children's positive experiences with childhood vaccination.

I think vaccines are very important. I recall in my country [Dominican Republic] when were kids our mother taking us to be vaccinated. There were people in communities who did not get vaccines and people died. So, I think vaccines are very important and I personally believe one should get all the vaccines that are important to prevent disease...

Furthermore, some fathers explicitly discussed vaccines as being lifesaving, and recalled stories of family members and friends who had experienced disabling or life-threatening effects of vaccine-preventable diseases such as poliomyelitis (poliovirus) and measles in their home countries.

Vaccine are important and especially for children to have all vaccines when they are little. I have a cousin who had paralysis when he was a child. For some reason his mother had missed the vaccination [campaign] back in El Salvador...I recall my mother always telling us when were scared [of vaccines] that it's better to hurt one day than to suffer a whole life...

\subsubsection{Perceived risks of the HPV vaccine}

When asked about any concerns about the HPV vaccine, a couple of fathers mentioned concerns about the vaccine having a negative effect on their daughters' sexual disinhibition, while a few fathers mentioned $(n=4)$ concerns about vaccine safety and side effects. For example, two fathers reported,

As fathers we worry about our children's health and safety. We have to keep vigilant, pay attention where the kids are, what they are doing... but my problem with the vaccine [HPV] for the girls is that nowadays they know more than us [parents] so I worry that they [girls] think they are safe [to have sex], you know what I mean?! I personally think this vaccine should be given when they [girls] are older...

One [parents] worries about negative effects [safety] of vaccines because nothing is 100\% safe. In the case of the papillomavirus vaccine my understanding is that it will do more benefits than harm. There is always a risk [adverse effect of vaccine]. But if you know it will have benefit for your child, as a parent you want to vaccinate...you need to trust the doctors because they are the ones who studied, who have the knowledge, right?

Another father mentioned,

I think vaccines are pretty safe. But there are lot of people who think that vaccine can cause harm to their children. Like, I heard some people say that vaccines cause autism in children and other problems. Personally, I don't think that is the case. I don't think it's the vaccine. Vaccines are tested and pretty safe. I never had any problems, and my children never had any problems after they got any vaccine...so, in my case, I am in favor of them getting vaccines...

3.2.4. Theme: High willingness to vaccinate against HPV if recommended by healthcare provider

3.2.4.1. Trust in healthcare providers and willingness to vaccinate if recommended

Across all interviews, all fathers expressed trust in their healthcare providers. Although most fathers spoke of mothers being the primary parent in contact with healthcare providers for the care of their children, nearly all fathers said that they trusted their healthcare providers and were supportive of their healthcare recommendations for their children including vaccination. For example, one father said: 
I trust their [children] doctor. They go to the same doctor now for many years and my wife really likes the doctor. She [doctor] studied and trained, so always when she [doctor] says they need a vaccine, or an exam, we always agree.

When asked specifically if they would be willing to vaccinate their children against HPV if recommended by their child's doctor, all fathers reported willingness to vaccinate both their daughters and their sons against HPV. One father said,

I think if it's very important the doctor should tell the parents, right? How would I know? The doctor is the one who studied all these years... I think if it's important the doctor should tell the parents and convince them. You know, give the information because it's not that the parents don't want to vaccinate but sometimes, we don't know about that there is a vaccine ... like when the kids are really little, they tell us, and my children got all the vaccines...but this one [HPV] I never heard about it.

\subsubsection{2: Healthcare provider recommendation single most important factor}

Across all interviews, fathers reported that if recommended by their child's primary healthcare provider, they would be supportive of vaccinating their children against HPV. This was true for both fathers who reported being aware of HPV and the HPV vaccine and those who were not aware of the HPV vaccine. These are illustrated in the following two quotes.

You know, there are some people who say vaccines sometimes are not safe, and that's not good to give too many vaccines because it can cause problems... But we [father and partner] always say that if the doctor is recommending and says it's important, we give them. We [parents] want them to be healthy and avoid diseases...

...One hears all sorts of things about vaccines and at the end it's your child's life and you will not trust just anyone or any information. In my case at least, I will tell you, I would trust my child's doctor. If her [daughter] doctor recommends the vaccine and explains that it's important, I will support her getting the vaccine $[H P V] . .$.

Only one father expressed hesitancy towards vaccinating his children against HPV due to concerns about potential vaccines' negative side-effects. This father mentioned having a family member, whose son had experienced vaccine side-effects,including getting "sick" after vaccination, which according to this father, led to the child developing autism.

To tell you the truth, I am not sure. One sometimes worries about the negative consequences of vaccines. Vaccines can be good, but they also have risks. One knows that vaccines are not $100 \%$ safe. There are risks. My nephew was a perfect child until he was about 1 and half years old, then all of a sudden, he started showing problems. We can't tell for sure but in his case, he might have suffered some bad effects of the vaccine...

\section{Discussion}

The majority of fathers participating in our study were foreign-born $(n=13)$, and the remainder $(n=6)$ were born and had lived in Puerto Rico (United States territory) for most of their lives. Almost two-thirds were classified as having low acculturation levels, and almost $85 \%$ reported Spanish as their primary language spoken with family and friends. The limited number of previous studies with Latino fathers suggest the influence of acculturation level, birthplace, and number of years lived in the United States on parents' willingness to vaccinate and acceptability of the HPV vaccine [35-37]. These studies found that parents born in the United States and those who had lived in the United States for more than 15 years were more likely to report having vaccinated their children against HPV than those who had recently moved to the United States [35-37].

Overall, our findings suggest that Latino fathers participating in our study had lower awareness and lower knowledge of HPV transmission, HPV-associated cancers, and the HPV vaccine. 
Nonetheless, fathers held positive attitudes towards vaccines in general, and nearly all reported increased willingness to vaccinate both their sons and daughters against the HPV if recommended by a healthcare provider. These important findings suggest opportunities for the promotion of the HPV vaccine among Latino fathers that have implications for cancer education programs targeting both fathers and healthcare providers. First, these findings suggest a need for increased awareness and education of Latino fathers about HPV and the HPV vaccine. A recent systematic review of studies ( $\mathrm{n}=11$ ) conducted in the United States exploring HPV knowledge, awareness, beliefs, attitudes, and acceptability of the HPV vaccine among Latino fathers found that overall fathers reported lower awareness and knowledge of HPV and the HPV vaccine compared to mothers [22]. Moreover, one of the 11 studies included in this review conducted exclusively with Latino fathers found that although fathers held positive attitudes toward the HPV vaccine, a notable number of participants were unsure of or had not formed an opinion about the HPV vaccine [24].

These finding support the results of previous studies conducted with other racial and ethnic minority groups [23,31,38-42] and Latinos [15-18,20,23-26] documenting the unique and crucial influence of healthcare providers on fathers' acceptability and willingness to vaccinate their children against HPV. Although the vast majority of fathers participating in our study reported that their child's mother was the primary parent taking children to healthcare visits, fathers also mentioned close communication with their wives and/or partners about their children's health visits. Combined, these findings suggest interventions designed to promote HPV among Latino fathers, and their families should aim not only to increase fathers' awareness and knowledge of HPV transmission, HPV-associated cancers, and the HPV vaccine, but also involve promotion of improved healthcare providers communication and counseling about the HPV vaccine with Latino parents including fathers. Future studies should explore the combination of both healthcare- and family-focused interventions to increased HPV vaccination among Latino parents [43-45].

Strengths of the present study include the heterogeneity of the study sample with Latino fathers of Caribbean, North, Central, and South American backgrounds. Similar themes emerged across subpopulation groups, which strengthen the study's findings. In addition, study team members are bilingual and bicultural, and immigrant Latinas. As with any research, there are also limitations to this study. The findings are based on a small, convenience sample of Spanish-speaking, majority immigrant Latino fathers with low acculturation levels. Therefore, these results may not be generalizable to English-speaking Latino fathers and those with higher acculturation levels. Participants were recruited from urban communities in Massachusetts, a state with higher rates of government-sponsored healthcare coverage. Therefore, results may also not be generalizable to other populations in the United States. Furthermore, the use of snowball sampling may have led to the selection of participants who share similar beliefs, attitudes, and experiences. Despite the use of a semi-structured interview instrument and interviewers' training, there is potential for interviewer bias.

\section{Conclusions}

In conclusion, the findings of the present study indicate the need for increased efforts to raise awareness and knowledge among Latino fathers of HPV and the HPV vaccine. The fact that fathers participating in this study reported a high willingness to vaccinate both their sons and daughters against HPV if recommended by their children's primary healthcare provider suggests opportunities for education interventions endorsed by healthcare providers to promote the HPV vaccination among Latino fathers and their families. Additionally, most fathers mentioned mothers as the primary parent directly interacting with healthcare providers and as informants of their children's healthcare visits, including vaccinations. These findings combined suggest that effective interventions aimed at promoting the HPV vaccination among Latino fathers and increasing Latino children's HPV vaccination rates may require a combined healthcare- and family-focused approach involving fathers' decision support for vaccinating their children against HPV. Given the limited research focused on Latino fathers, the study's findings are valuable in building a knowledge foundation needed for developing future studies and interventions targeting Latino fathers living in 
the United States. Future research should quantify Latino fathers' awareness, knowledge, and acceptability of the HPV vaccine for their sons and daughters, and preferences for educational interventions to promote HPV vaccination among Latino fathers. This information will be relevant to the development of interventions tailored to meet the needs of Latino parents and ultimately increase their children's HPV vaccination rates.

Supplementary Materials: The following are available online at www.mdpi.com/xxx/s1, Table S1: In-Depth Interview Guide Latino Parents HPV Project-UMB; Table S2: Demographic characteristics of the sample.

Author Contributions: Conceptualization, A.C.L.; methodology, A.C.L.; formal analysis, A.C.L., D.D.; investigation, A.C.L., M.J.V., D.D., P.G.; resources, A.C.L.; data curation, A.C.L., data collection, D.D., M.J.V.; writing-original draft preparation, A.C.L.; writing-review and editing, A.C.L., P.G.; supervision, A.C.L; project administration, A.C.L.; funding acquisition, A.C.L., M.J.V. All authors have read and agreed to the published version of the manuscript.

Funding: This research was funded by faculty funds from the University of Massachusetts Boston (Ana Cristina Lindsay). Madelyne J. Valdez participated in this research as part of a summer internship (Dr. Ana Cristina Lindsay, Mentor) awarded by the University of Massachusetts Boston-Dana-Farber/Harvard Cancer Center Partnership for Advancing Cancer Health Equity funded by the National Cancer Institute (NCI 2U54CA156734-06A1). The findings and conclusions in this report are those of the authors and do not necessarily represent the official position of the University of Massachusetts Boston-Dana-Farber/Harvard Cancer Center Partnership for Advancing Cancer Health Equity or the National Cancer Institute.

Acknowledgments: The authors thank the fathers that participated in the study as well as community-based agencies for support in recruitment efforts.

Conflicts of Interest: The authors declare no conflict of interest.

\section{References}

1. Markowitz, L. E., Gee, J., Chesson, H., \& Stokley, S. Ten Years of Human Papillomavirus Vaccination in the United States. Academic pediatrics. 2020, 18(2S), S3-S10. https://doi.org/10.1016/j.acap.2017.09.014

2. Walker TY, Elam-Evans LD, Yankey D, et al. National, Regional, State, and Selected Local Area Vaccination Coverage Among Adolescents Aged 13-17 Years - United States, 2018. MMWR Morb Mortal Wkly Rep. 2019, 68:718-723. DOI: http://dx.doi.org/10.15585/mmwr.mm6833a2

3. Jemal, A., Simard, E. P., Dorell, C., Noone, A. M., Markowitz, L. E., Kohler, B., Eheman, C., Saraiya, M., Bandi, P., Saslow, D., Cronin, K. A., Watson, M., Schiffman, M., Henley, S. J., Schymura, M. J., Anderson, R. N., Yankey, D., \& Edwards, B. K. Annual Report to the Nation on the Status of Cancer, 1975-2009, featuring the burden and trends in human papillomavirus (HPV)-associated cancers and HPV vaccination coverage levels. Journal of the National Cancer Institute. 2013, 105(3), 175-201. https://doi.org/10.1093/jnci/djs491

4. Bednarczyk, R. A., Ellingson, M. K., \& Omer, S. B. Human Papillomavirus Vaccination Before 13 and 15 Years of Age: Analysis of National Immunization Survey Teen Data. The Journal of infectious diseases. 2019, 220(5), 730-734. https://doi.org/10.1093/infdis/jiy682

5. Healthy People 2020 [Internet]. Washington, DC: U.S. Department of Health and Human Services, Office of Disease Prevention and Health Promotion [Accessed April 27, 2020]. Available from: [https://www.healthypeople.gov/2020/about-healthy-people/how-to-usehealthypeople.gov/frequently-asked-questions].

6. Brewer, N. T., \& Fazekas, K. I. Predictors of HPV vaccine acceptability: a theory-informed, systematic review. Preventive medicine. 2007, 45(2-3), 107-114. https://doi.org/10.1016/j.ypmed.2007.05.013

7. Adjei Boakye, E., Tobo, B. B., Rojek, R. P., Mohammed, K. A., Geneus, C. J., \& Osazuwa-Peters, N. Approaching a decade since HPV vaccine licensure: Racial and gender disparities in knowledge and awareness of HPV and HPV vaccine. Human vaccines \& immunotherapeutics. 2017, 13(11), 2713-2722. https://doi.org/10.1080/21645515.2017.1363133

8. Reimer, R. A., Schommer, J. A., Houlihan, A. E., \& Gerrard, M. Ethnic and gender differences in HPV knowledge, awareness, and vaccine acceptability among White and Hispanic men and women. Journal of community health. 2014, 39(2), 274-284. https://doi.org/10.1007/s10900-013-9773-y 
9. Daley, E. M., Marhefka, S., Buhi, E., Hernandez, N. D., Chandler, R., Vamos, C., Kolar, S., Wheldon, C., Papenfuss, M. R., \& Giuliano, A. R. Ethnic and racial differences in HPV knowledge and vaccine intentions among men receiving HPV test results. Vaccine. 2011, 29(23), 4013-4018. https://doi.org/10.1016/j.vaccine.2011.03.060

10. Netfa, F., Tashani, M., Booy, R., King, C., Rashid, H., \& Skinner, S. R. Knowledge, Attitudes and Perceptions of Immigrant Parents Towards Human Papillomavirus (HPV) Vaccination: A Systematic Review. Tropical medicine and infectious disease, 5(2), 58. https://doi.org/10.3390/tropicalmed5020058

11. Dempsey, A. F., Zimet, G. D., Davis, R. L., \& Koutsky, L. Factors that are associated with parental acceptance of human papillomavirus vaccines: a randomized intervention study of written information about HPV. Pediatrics. 2006, 117(5), 1486-1493. https://doi.org/10.1542/peds.2005-1381

12. Pew Research Center. How the U.S. Hispanic population is changing. Pew Research Center. 2017. https:/www.pewresearch.org/fact-tank/2017/09/18/how-the-u-s-hispanic-population-is-changing/

13. Martinez Tyson, D., Medina-Ramirez, P., Flores, A. M., Siegel, R., \& Aguado Loi, C. Unpacking Hispanic Ethnicity-Cancer Mortality Differentials Among Hispanic Subgroups in the United States, 2004-2014. Frontiers in public health. 2018, 6, 219. https://doi.org/10.3389/fpubh.2018.00219

14. Bodson, J., Warner, E. L., \& Kepka, D. Moderate Awareness and Limited Knowledge Relating to Cervical Cancer, HPV, and the HPV Vaccine Among Hispanics/Latinos in Utah. Health promotion practice. 2016, 17(4), 548-556. https://doi.org/10.1177/1524839916640271

15. Colón-López, V., Toro-Mejías, L.M.D., Conde-Toro, A., Serra-Rivera, M.J., Martínez, T.M., Rodríguez, V., ... Villanueva, H. Views on HPV and HPV Vaccination: The Experience at a Federal Qualified Clinic in Puerto Rico. Journal of Health Care for the Poor and Underserved. 2016, 27(3), 1411-1426. doi:10.1353/hpu.2016.0126

16. Victory, M., Do, T., Kuo, Y. F., \& Rodriguez, A. M. Parental knowledge gaps and barriers for children receiving human papillomavirus vaccine in the Rio Grande Valley of Texas. Human vaccines $\mathcal{E}$ immunotherapeutics. 2019, 15(7-8), 1678-1687. https://doi.org/10.1080/21645515.2019.1628551

17. Morales-Campos, D. Y., Snipes, S. A., Villarreal, E. K., Crocker, L. C., Guerrero, A., \& Fernandez, M. E. Cervical cancer, human papillomavirus (HPV), and HPV vaccination: exploring gendered perspectives, knowledge, attitudes, and cultural taboos among Mexican American adults. Ethnicity $\mathcal{E}$ health. 2018, 1-19. Advance online publication. https://doi.org/10.1080/13557858.2018.1494821

18. Warner, E. L., Lai, D., Carbajal-Salisbury, S., Garza, L., Bodson, J., Mooney, K., \& Kepka, D. Latino Parents' Perceptions of the HPV Vaccine for Sons and Daughters. Journal of community health. 2015, 40(3), 387-394. https://doi.org/10.1007/s10900-014-9949-0

19. Vanslyke, J. G., Baum, J., Plaza, V., Otero, M., Wheeler, C., \& Helitzer, D. L. HPV and cervical cancer testing and prevention: knowledge, beliefs, and attitudes among Hispanic women. Qualitative health research. 2008, 18(5), 584-596. https://doi.org/10.1177/1049732308315734

20. Roncancio, A. M., Carmack, C. C., Ward, K. K., Vernon, S. W., Muñoz, B. T., Cano, M. A., \& Cribbs, F. L. Toward a Model of HPV Vaccine Series Completion in Adolescent Hispanic Males: Identifying Mothers' Salient Behavioral, Normative, and Control Beliefs. Family \& community health. 2019, 42(2), 161-169. https://doi.org/10.1097/FCH.0000000000000221

21. Parra-Medina, D., Morales-Campos, D. Y., Mojica, C., \& Ramirez, A. G. Promotora Outreach, Education and Navigation Support for HPV Vaccination to Hispanic Women with Unvaccinated Daughters. Journal of cancer education: the official journal of the American Association for Cancer Education. 2015, 30(2), 353-359. https://doi.org/10.1007/s13187-014-0680-4

22. Suárez, P., Wallington, S. F., Greaney, M. L., \& Lindsay, A. C. Exploring HPV Knowledge, Awareness, Beliefs, Attitudes, and Vaccine Acceptability of Latino Fathers Living in the United States: An Integrative Review. Journal of community health. 2019, 44(4), 844-856. https://doi.org/10.1007/s10900019-00636-7

23. Galbraith, K. V., Lechuga, J., Jenerette, C. M., Moore, L. A., Palmer, M. H., \& Hamilton, J. B. Parental acceptance and uptake of the HPV vaccine among African Americans and Latinos in the United States: A literature review. Social science $\mathcal{E}$ medicine (1982). 2016, 159, 116-126. https://doi.org/10.1016/j.socscimed.2016.04.028

24. Kornfeld, J., Byrne, M. M., Vanderpool, R., Shin, S., \& Kobetz, E. HPV knowledge and vaccine acceptability among Hispanic fathers. The journal of primary prevention. 2013, 34(1-2), 59-69. https://doi.org/10.1007/s10935-013-0297-0 
25. Lindsay, A. C., Pineda, J. A., Valdez, M. J., Torres, M. I., \& Granberry, P. J. Central American Immigrant Parents' Awareness, Acceptability, and Willingness to Vaccinate Their Adolescent Children Against Human Papillomavirus: A Pilot Cross-Sectional Study. International journal of environmental research and public health. 2020, 17(8), 2869. https://doi.org/10.3390/ijerph17082869

26. Perkins, R. B., Mehta, P. K., \& Langrish, S. M. Fathers' intentions to accept human papillomavirus vaccination for sons and daughters: exploratory findings from rural Honduras. International journal of public health. 2012, 57(1), 143-148. https://doi.org/10.1007/s00038-011-0271-7

27. Faugier, J., \& Sargeant, M. Sampling hard to reach populations. Journal of advanced nursing. 1997, 26(4), 790-797. https://doi.org/10.1046/j.1365-2648.1997.00371.x

28. Patton, M. Q. Qualitative research \& evaluation methods (3rd ed.). Thousand Oaks, CA: Sage. 2001.

29. Lindsay, A. C., Delgado, D., Valdez, M. J., Restrepo, E., \& Guzman, Y. M. "I don't Think He Needs the HPV Vaccine Cause Boys Can't Have Cervical Cancer": a Qualitative Study of Latina Mothers' (Mis) Understandings About Human Papillomavirus Transmission, Associated Cancers, and the Vaccine. Journal of cancer education: the official journal of the American Association for Cancer Education. 2020 10.1007/s13187-020-01824-z. Advance online publication. https://doi.org/10.1007/s13187-020-01824-z

30. Lindsay, A. C., Delgado, D., Valdez, M. J., Restrepo, E., Guzman, Y. M, \& Granberry, P. Acceptance of the HPV vaccine in a multi-ethnic sample of Latinx mothers. Qualitative health research. 2020, 10.1177/10497323. Advance online publication. https://doi.org/ 10.1177/10497323

31. Katz, I. T., Bogart, L. M., Fu, C. M., Liu, Y., Cox, J. E., Samuels, R. C., Chase, T., Schubert, P., \& Schuster, M. A. Barriers to HPV immunization among blacks and Latinos: a qualitative analysis of caregivers, adolescents, and providers. BMC public health. 2016, 16(1), 874. https://doi.org/10.1186/s12889-016-35294

32. Marin, G., Sabogal, F., Marin, B.V., Otero-Sabogal, G., Perez-Stable, E.J. Development of a short acculturation scale for Hispanics. Hisp J Behav Sci. 1987, 9:183-205. 10.1177/07399863870092005.

33. Ellison, J., Jandorf, L., \& Duhamel, K. Assessment of the Short Acculturation Scale for Hispanics (SASH) among low-income, immigrant Hispanics. Journal of cancer education: the official journal of the American Association for Cancer Education. 2011, 26(3), 478-483. https://doi.org/10.1007/s13187-011-0233$\mathrm{Z}$

34. Braun, V., \& Clarke, V. Using thematic analysis in psychology. Qualitative Research in Psychology. 2006, 3(2), 77-101. https://doi.org/10.1191/1478088706qp063oa

35. Colón-López, V., Toro-Mejías, D., Conde-Toro, L. M., A., et al.. Views on HPV and HPV vaccination: The experience at a federal qualified clinic in Puerto Rico. Journal of Health Care for the Poor and Underserved. 2016 27(3), 1411. https://doi.org/10.1353/ hpu.2016.0126. 29.

36. Kepka, D., Ding, Q., Bodson, J., et al.. Latino parents' awareness and receipt of the HPV vaccine for sons and daughters in a state with low three-dose completion. Journal of Cancer Education. 2015 30(4), 808-812. https://doi.org/10.1007/s1318 7-014-0781-0.

37. Kepka, D., Warner, E. L., Kinney, A. Y., et al.. Low human papillomavirus (HPV) vaccine knowledge among Latino parents in Utah. Journal of Immigrant and Minority Health. 2015, 17(1), 125-131. https://doi.org/10.1007/s10903-014-0003-1

38. Miyoshi, A., Takiuchi, T., \& Kimura, T. HPV vaccination in Japan: can educational intervention promote a father's intention to encourage his daughter's vaccination?. International journal of clinical oncology. 2020, 25(4), 746-754. https://doi.org/10.1007/s10147-019-01575-y

39. Thomas, T. L., Strickland, O. L., \& Higgins, M. Mothers, Fathers, Sons, and Human Papillomavirus Immunization Practices. Family \& community health. 2017, 40(3), 278-287. https://doi.org/10.1097/FCH.0000000000000104

40. Mupandawana, E. T., \& Cross, R. Attitudes towards human papillomavirus vaccination among African parents in a city in the north of England: a qualitative study. Reproductive health. 2016, 13(1), 97. https://doi.org/10.1186/s12978-016-0209-x

41. Hanley SJ, Yoshioka E, Ito Y, et al. An exploratory study of Japanese fathers' knowledge of and attitudes towards HPV and HPV vaccination: does marital status matter? Asian Pac J Cancer Prev. 2014, 15(4):1837-1843. doi:10.7314/apjcp.2014.15.4.1837

42. Kuznetsov, L., Zippel, S.A., Ruzicka, T., Kuznetsov, A. Fathers' knowledge of and attitude towards human papillomavirus infection, genitoanal warts, cervical cancer and HPV vaccine. Int J Public Health. 2012, (57), 651-653. https://doi.org/10.1007/s00038-012-0351-3 
43. Fiks, A. G., Grundmeier, R. W., Mayne, S., Song, L., Feemster, K., Karavite, D., Hughes, C. C., Massey, J., Keren, R., Bell, L. M., Wasserman, R., \& Localio, A. R. Effectiveness of decision support for families, clinicians, or both on HPV vaccine receipt. Pediatrics. 2013, 131(6), 1114-1124. https://doi.org/10.1542/peds.2012-3122

44. Niccolai, L. M., \& Hansen, C. E.. Practice- and Community-Based Interventions to Increase Human Papillomavirus Vaccine Coverage: A Systematic Review. JAMA pediatrics. 2015, 169(7), 686-692. https://doi.org/10.1001/jamapediatrics.2015.0310

45. Leung, S., Akinwunmi, B., Elias, K. M., \& Feldman, S. Educating healthcare providers to increase Human Papillomavirus (HPV) vaccination rates: A Qualitative Systematic Review. Vaccine: X. 2019, 3 , 100037. https://doi.org/10.1016/j.jvacx.2019.100037 